pgRNA under LLD in HBeAg-positive patients was significantly lower than that in HBeAg-negative patients $(15.75 \%$ [23/46] vs.77.59\%[45/58], $P<0.001)$. Overall, serum HBV pgRNA strongly correlated with $\mathrm{HBcrAg}(r=0.760, P<0.001)$, and moderately correlated with HBV DNA $(r=0.663$, $P<0.001)$ and HBsAg $(r=0.670, P<0.001)$. As compared to HBsAg and HBV DNA, only HBcrAg showed stable correlation with serum HBV pgRNA both in HBeAg-positive and HBeAg-negative patients. Multivariate logistic regression analysis showed that only $\mathrm{HBcrAg}$ was independently associated with serum HBV pgRNA below LLD (OR: 2.900, $P=0.000$ ) (AUROC $=0.944$, cut-off value $=6.60 \log 10 \mathrm{U} / \mathrm{mL}$ ).

Conclusions Serum HBV pgRNA level differed between HBeAg-positive and HBeAg-negative patients; and it had a better and more stable correlation with serum HBcrAg than serum HBV DNA and HBsAg, irrespective of HBeAg status. Serum HBcrAg was an independent predictor of serum $\mathrm{HBV}$ pgRNA below LLD.

\section{IDDF2019-ABS-0340 THE SPONTANEOUS SYMPTOMATIC ACUTE EXACERBATION OF CHRONIC HEPATITIS B (CHBAE) - CLINICAL PROFILE AND OUTCOMES WITH EARLY ANTI-VIRAL THERAPY}

${ }^{1}$ Gaurav Padia*, ${ }^{2}$ Ajay Kumar, ${ }^{3}$ Amol Dahale, ${ }^{4}$ Siddharth Srivastava. ${ }^{1}$ Senior Resident, GB Pant Institute of Postgraduate Medical Education and Research, New Delhi, India; ${ }^{2}$ Associate Professor, GB Pant Institute of Postgraduate Medical Education and Research, New Delhi, India; ${ }^{3}$ Assistant Professor, GB Pant Institute of Postgraduate Medical Education and Research, New Delhi, India; ${ }^{4}$ Professor, GB Pant Institute of Postgraduate Medical Education and Research, New Delhi, India

\subsection{6/gutjnl-2019-IDDFabstracts.309}

Background Spontaneous severe symptomatic reactivation of chronic hepatitis $\mathrm{B}$ (CHBAE) has high mortality once patient decompensates. The aim of the study was to evaluate the outcome with early anti-viral therapy (AVT) in CHBAE.

Methods Consecutive adult patients having CHBAE seen at the Department of Gastroenterology, G.I.P.M.E.R.New Delhi,

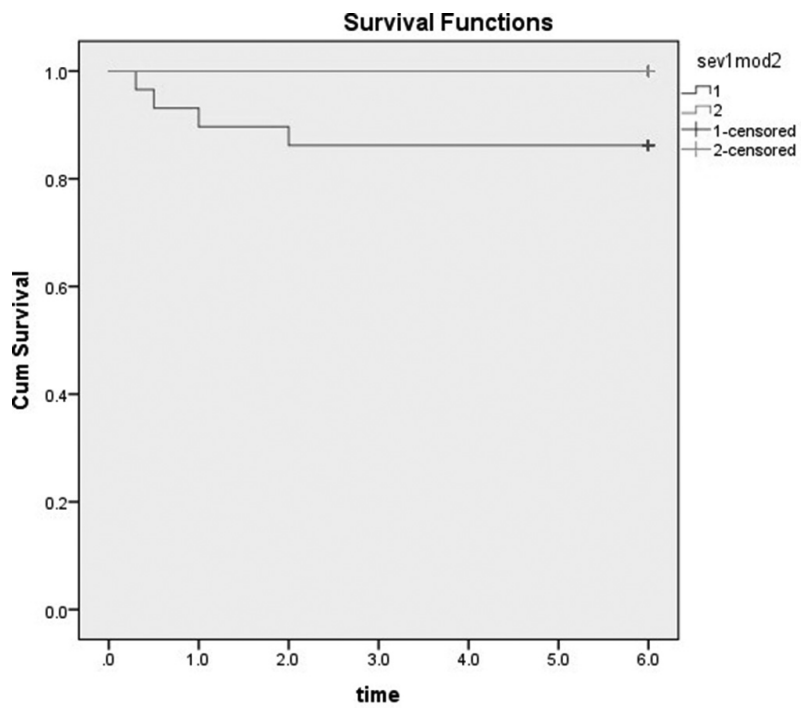

Abstract IDDF2019-ABS-0340 Figure 1
India were enrolled in the study. CHBAE severity was classified on basis of clinical features, total bilirubin \& prolongation of prothrombin time as mild (Bil 2-5 mg/dl, PT $<3$ secs), moderate(Bil $>5-15 \mathrm{mg} / \mathrm{dl}$, PT $3-5$ secs \& severe (Bil $>15 \mathrm{mg} / \mathrm{dl}$ or PT $>5$ secs or acute decompensation i.e. ascites $\&$ or hepatic encephalopathy). AVT (Tenofovir or Entecavir) was started in moderate $\&$ severe disease at the time of initial presentation (i.e Early AVT). Patients were followed at weekly interval till improvement \& then every month till 3 months. The primary outcome was survival at 3 months.

Results 53 patients with confirmed CHBAE finally included. Mean age was $39.8 \pm 14.4$ yrs. \& 38(72\%) were males. Mild $\operatorname{CHBAE}(n=3)$, moderate $\operatorname{CHBAE}(n=21) \&$ severe $\operatorname{CHBAE}(n=29)$ cases respectively. Moderate group had (cirrhotic $\mathrm{n}=03$ ) \& median duration of symptoms was 25 days (21-28 days), S. Bil $9.3 \mathrm{mg} / \mathrm{dl}(7.9-10.9 \mathrm{mg} / \mathrm{dl})$, ALT 1161 IU/ml (608-1395 IU/ml), S. Alb $3.9 \mathrm{gm} / \mathrm{dl}(3.6-4.4 \mathrm{gm} / \mathrm{dl})$ \& HBV DNA $33910 \mathrm{IU} / \mathrm{ml}$ (7916-1900000 IU/ml) respectively. Severe CHBAE group had (cirrhotic $n=11$ ) \& median duration of symptoms was 28 days (21-42 days), S. Bil $19.32 \mathrm{mg} /$ dl (15.4-23.1 mg/dl), ALT $1088 \mathrm{IU} / \mathrm{ml}$ (311-1440 IU/ml), S. Alb $3.5 \mathrm{gm} / \mathrm{dl}(3.1-4.2 \mathrm{gm} / \mathrm{dl})$ \& HBV DNA was $174931 \mathrm{IU} /$ $\mathrm{ml}(7600$ - $667601 \mathrm{IU} / \mathrm{ml})$. In moderate CHBAE (Entecavir $\mathrm{n}=14$, Tenofovir $\mathrm{n}=7$ ) \& in severe CHBAE (Entecavir $\mathrm{n}=24$, Tenofovir $\mathrm{n}=05$ ) were treated respectively. There were 4 $(7.5 \%)$ deaths in severe CHBAE, all deaths were in cirrhotic patients whom presented with decompensation. (figure 1)

Conclusions Severe CHBAE presents with decompensation of underlying cirrhosis \& early initiation of AVT at initial presentation in symptomatic moderate \& severe CHBAE prevents clinical deterioration, improves prognosis and reduces mortality.

\section{IDDF2019-ABS-0345 SARCOPENIA IN CIRRHOSIS: A RISK FACTOR FOR HOSPITALIZATIONS AND SHORT TERM MORTALITY}

${ }^{1}$ Surakshith Thyloor K*, ${ }^{1}$ Mandhir Kumar, ${ }^{1}$ Piyush Ranjan, ${ }^{2}$ Samarjit Ghuman, ${ }^{1}$ Anil Arora. ${ }^{1}$ Institute of Liver, Gastroenterology, and Pancreaticobiliary Sciences, Sir Ganga Ram Hospital, New Delhi, India; '2Department of Radiodiagnosis, Sir Ganga Ram Hospital, New Delhi, India

\subsection{6/gutjnl-2019-IDDFabstracts.310}

Background Sarcopenia is an under-recognized complication of cirrhosis which negatively impacts survival and quality of life. There is a paucity of Indian data regarding the prevalence and clinical significance of sarcopenia in cirrhotics. Our aim was to assess the prevalence of sarcopenia and its impact on short-term morbidity and mortality (6 months) of patients with cirrhosis.

\begin{tabular}{llll} 
Abstract IDDF2019-ABS-0345 Table1 & & \\
\hline Characteristics & $\begin{array}{l}\text { No sarcopenia } \\
(\mathrm{N}=82)\end{array}$ & $\begin{array}{l}\text { Sarcopenia } \\
(\mathrm{N}=74)\end{array}$ & P-value \\
\hline Ascites & 49 & 64 & 0.003 \\
Encephalopathy & 5 & 28 & 0.0001 \\
Re-admission & 18 & 55 & 0.0001 \\
Death & 3 & 18 & 0.0002 \\
\hline
\end{tabular}


Methods This was a prospective study of 156 consecutive patients with liver cirrhosis. The cross-sectional area of the right psoas muscle was measured at the level of L3-vertebra and Psoas muscle index (PMI) $\left(\mathrm{mm}^{2} / \mathrm{m}^{2}\right)$ was calculated. Sarcopenia was defined based on the CT study of patients with irritable bowel syndrome (controls), as PMI less than $295 \mathrm{~mm}^{2}$ / $\mathrm{m}^{2}$ and $356 \mathrm{~mm}^{2} / \mathrm{m}^{2}$ for females and males respectively.

Results 74(47.4\%) patients had sarcopenia. Sarcopenia was more common in males and patients with alcohol-related liver disease(ALD). $70 \%$ of ALD patients had sarcopenia. There was a negative correlation between the PMI and severity of liver disease as assessed by Child and MELD scores $(r=-$ 0.591 and -0.465 respectively). Patients with encephalopathy, ascites and coagulopathy had a higher prevalence of sarcopenia (table1).

$55(74.3 \% \mathrm{v} / \mathrm{s} 22 \%)$ of the patients who had sarcopenia required readmission within 6 months of follow-up as compared to those without sarcopenia $(p=0.0001)$ (figure 1$)$. 6months mortality was higher among patients with sarcopenia (24.3\% v/s 3.7\%; p=0.002). MELD score and PMI were independently associated with higher mortality. PMI Cut off value for predicting mortality obtained was $305.9 \mathrm{~mm} 2 /$ $\mathrm{m} 2$ with a sensitivity of $76.2 \%$ and a false positivity of $22.2 \%$. (AUC was 0.805; 95\% confidence interval:0.69-0.91, $\mathrm{p}=0.001)$.

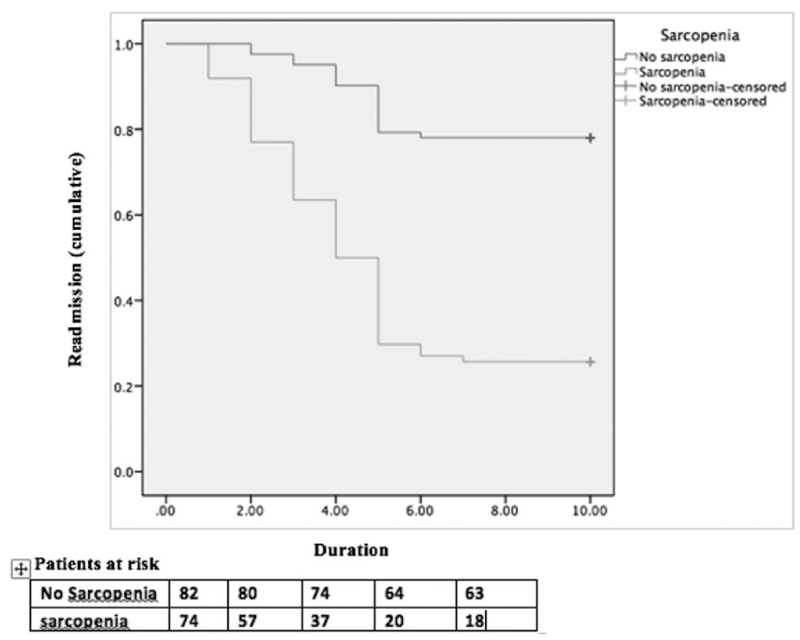

Abstract IDDF2019-ABS-0345 Figure 1 Risk of readmission among sarcopenic and non-sarcopenic patients

Conclusions PMI for the assessment of sarcopenia is simple, objective and reproducible. Sarcopenia is seen in about half of the patients with liver cirrhosis and more common in patients with alcohol-related liver disease, ascites and encephalopathy. Sarcopenic patients have frequent hospitalizations, lower shortterm survival and worse prognosis. 